\title{
Risk management in clinical practice. Part 10. Periodontology
}
IN BRIEF
- Failure to diagnose or treat periodontal disease is the fastest growing area of litigation in dentistry.
- Record keeping is the most essential part of managing periodontal disease.
- Radiographs are a necessary part of assessing periodontal disease and its progress.
- Patients must be made aware of factors that influence the outcome of periodontal disease treatment.

\section{A sizeable proportion of patients in clinical practice will have some form of periodontal disease and most of these patients can be well managed in primary care. Unfortunately, dento-legal claims regarding inappropriate periodontal care are increasing rapidly and are now one of the most common reasons for litigation in dentistry. In this paper we will look at aspects of contemporary management of periodontal disease in clinical practice and offer guidance for examination, management and referral.}

\section{PERIODONTITIS - \\ A SILENT DISEASE}

Up to $90 \%$ of the population will experience some attachment loss during their life. Of these, approximately $25 \%$ will lose one or more teeth as a result of periodontitis. Unfortunately, until the point of facing

\begin{tabular}{l} 
RISK MANAGEMENT \\
IN CLINICAL PRACTICE \\
\hline 1. Introduction \\
2. Getting to 'yes' - the matter of consent \\
3. Crowns and bridges \\
4. Endodontics \\
5. Ethical considerations for dental \\
enhancement procedures \\
6a. Identifying and avoiding medico-legal \\
risks in complete denture prosthetics \\
66. Identifying and avoiding medico-legal \\
risks in removable dentures \\
7. Dento-legal aspects of orthodontic \\
practice \\
8. Temporomandibular disorders \\
9. Dental implants \\
10. Periodontology \\
11. Oral surgery
\end{tabular}

${ }^{1 *}$ Specialist in Periodontics, PerioLondon, 4 Queen Anne Street, London, W1G 9ZF; ${ }^{2}$ Specialist in Periodontics, Perio London, 4 Queen Anne Street, London, W1G 9ZF/ Professor of Restorative Dentistry and Evidence-Based Healthcare, Unit of Periodontology, UCL Eastman Dental Institute, 256 Gray's Inn Road, London, WC1X 8LD *Correspondence to: Dr Paul Baker Email:paul@periolondon.co.uk

\section{Refereed Paper}

Accepted 20 October 2009

DOI: 10.1038/sj.bdj.2010.1084

British Dental Journal 2010; 209: 557-565 tooth loss, many people will be unaware that they have had periodontal disease for some years. As a result, irreversible damage will have occurred which could have been predictably prevented or treated if identified at an early stage.

The aggressive forms of periodontal disease may result in significant amounts of attachment loss occurring at a young age or in a relatively short timeframe. In such cases, early diagnosis and proper management are essential.

Periodontal dento-legal claims frequently involve a failure to diagnose the disease, or a failure to adequately treat it. In this article we will set out guidance to help develop systems for effective screening, planning of periodontal prevention and treatment. However, this is not a textbook on periodontology and more detailed aspects will be found in textbooks such as Palmer \& Floyd. ${ }^{1}$

\section{DIAGNOSIS}

As with any disease, proper management starts with a correct diagnosis. Patients attending a dental check-up have a right to expect that they are receiving a thorough examination of the mouth, teeth and supporting structures, not just dental decay. Figure 1 shows periapical radiographs of a 55-year-old patient who reports being a regular dental attender throughout his adult life. He presents with advanced horizontal bone loss and in areas the bone loss is approaching the apices of teeth. There is general mobility and teeth have been recently removed because of this. His dentist had never informed him of his periodontal disease. Unfortunately this is not an uncommon picture in patients being referred for specialist treatment within the UK. Nothing can justify such a lack of basic care.

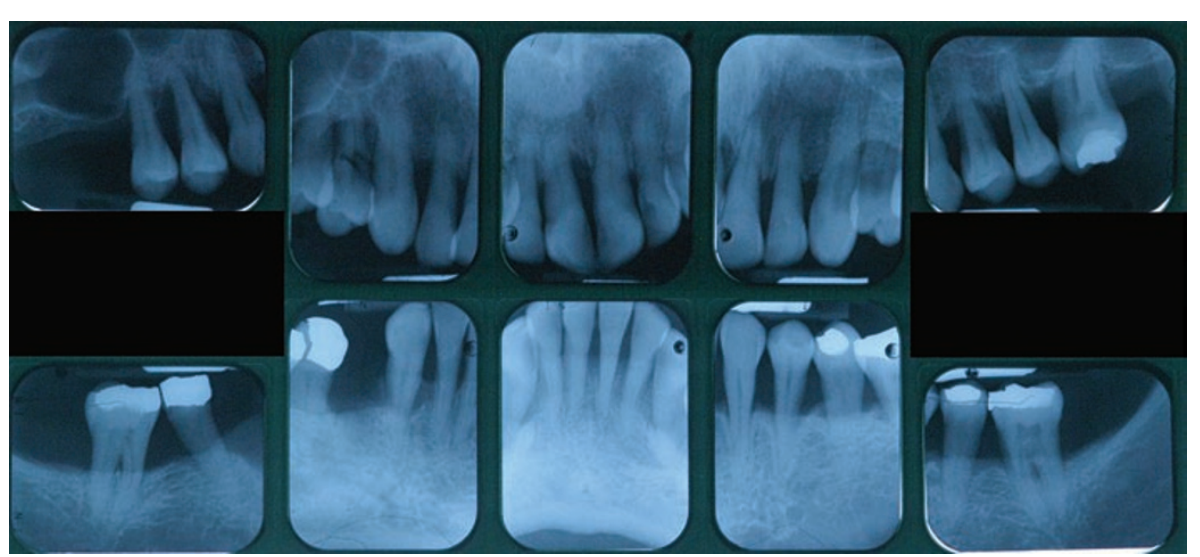

Fig. 1 Periapical radiograph set of a 55-year-old patient, showing advanced horizontal bone loss 


\section{Consequences of failure to diagnose}

The chronic periodontal conditions are mostly asymptomatic until they reach the later stages of the disease. Symptoms of periodontal disease that may cause a patient to seek advice from a dentist, such as mobility of the teeth, drifting or pain from a periodontal abscess, are all associated with advanced bone loss. Treatment at this stage is usually complex, and indeed extraction may be the only option. In contrast, the management of early periodontitis is relatively straightforward and predictable. It should therefore be obvious that early diagnosis is extremely important in the management of periodontal disease.

\section{Relevant dental and medical history}

As with all diagnosis, the initial presenting complaint and concerns of the patient should be ascertained. This may give the clinician the first hint of what to expect when it comes to the diagnosis. As already mentioned, patient symptoms tend to occur late in the progression of periodontal disease, so the absence of symptoms is no excuse for failure to screen for periodontal disease. The patient's concerns should also be taken into account when formulating the treatment plan. Figure 2, for example, shows a patient whose presenting complaint is the drifted and over-erupted upper left central incisor. This is a result of undiagnosed and untreated periodontal disease. Treatment of the active periodontal disease alone will not correct this, indeed the associated recession may increase the aesthetic concern for the patient. Achieving periodontal health might be fundamental to addressing the problem, or alternative treatment, such as extraction and replacement, may better suit the patient. Defining treatment aims and clarifying what can be

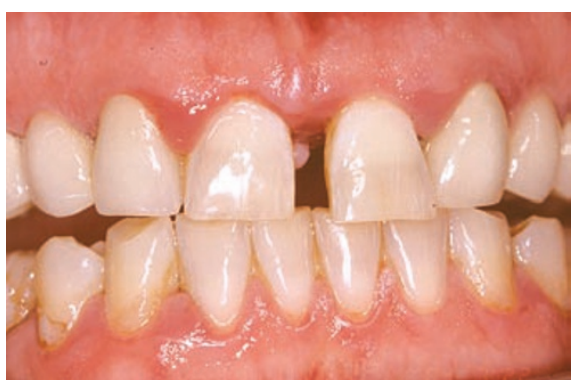

Fig. 2 Drifted and over-erupted upper left central incisor as a result of undiagnosed and untreated periodontal disease

\begin{tabular}{|c|c|c|c|}
\hline Code & Clinical features & Represents & Management \\
\hline Code 0 & $\begin{array}{l}\text { No pockets exceeding } 3 \mathrm{~mm} \\
\text { No plaque retentive factors } \\
\text { No bleeding after gentle probing }\end{array}$ & Health & None \\
\hline Code 1 & $\begin{array}{l}\text { No pockets exceeding } 3 \mathrm{~mm} \\
\text { No plaque retentive factors } \\
\text { Bleeding after gentle probing }\end{array}$ & Gingivitis & Oral hygiene instruction \\
\hline Code 2 & $\begin{array}{l}\text { No pockets exceeding } 3 \mathrm{~mm} \\
\text { Plaque retentive factors present (such } \\
\text { as calculus or overhanging margins) }\end{array}$ & $\begin{array}{l}\text { Gingivitis with sec- } \\
\text { ondary local factor }\end{array}$ & $\begin{array}{l}\text { Removal of local factors } \\
\text { Oral hygiene instruction }\end{array}$ \\
\hline Code 3 & $\begin{array}{l}\text { Periodontal probing of } 4-5 \mathrm{~mm} \text { (black } \\
\text { band of WHO probe partially visible) on } \\
\text { one or more sites }\end{array}$ & $\begin{array}{l}\text { Early chronic } \\
\text { periodontitis }\end{array}$ & $\begin{array}{l}\text { Periodontal probing } \\
\text { depths recorded for the } \\
\text { affected sextants } \\
\text { Appropriate level of } \\
\text { periodontal treatment }\end{array}$ \\
\hline Code 4 & $\begin{array}{l}\text { Periodontal probing of } 6 \mathrm{~mm} \text { or more } \\
\text { (black band of WHO probe disappears } \\
\text { into pocket) on one or more site }\end{array}$ & $\begin{array}{l}\text { Moderate to } \\
\text { advanced chronic } \\
\text { periodontitis }\end{array}$ & $\begin{array}{l}\text { Full periodontal } \\
\text { assessment } \\
\text { Appropriate level of } \\
\text { periodontal treatment }\end{array}$ \\
\hline Code $^{*}$ & $\begin{array}{l}\text { Attachment loss (probing depth plus } \\
\text { recession) of } 7 \mathrm{~mm} \text { or more, or if a } \\
\text { furcation can be probed }\end{array}$ & $\begin{array}{l}\text { Moderate to } \\
\text { advanced chronic } \\
\text { periodontitis or a site } \\
\text { requiring complex } \\
\text { periodontal treatment }\end{array}$ & $\begin{array}{l}\text { Full periodontal } \\
\text { assessment } \\
\text { Appropriate level of } \\
\text { periodontal treatment }\end{array}$ \\
\hline
\end{tabular}

achieved will help to select the appropriate direction for treatment and avoid patient dissatisfaction later.

Questions regarding the previous dental history should be asked, and, where it is thought to be relevant, these should include any previous periodontal treatment. While assumptions cannot be made about a lack of response to treatment in the past, treatment planning decisions may be altered in the context of success or failure of previous treatment. A family history of periodontal disease or early tooth loss may also be relevant and indicate a patient with a higher risk of susceptibility.

A comprehensive medical history should be taken for all patients and be kept upto-date. Of particular interest to the periodontist is the smoking history, both past and present. There is also an increased susceptibility to periodontal attachment loss in patients with poorly controlled diabetes. Since Type II diabetes has a genetic link, enquiring about a family history may be helpful in identifying borderline cases. Periodontal procedures that involve subgingival instrumentation, including periodontal probing, require antibiotic prophylaxis in patients who are at risk of infective endocarditis. Recent changes in the guidelines have significantly reduced the number of patients to whom this applies. ${ }^{2}$

\section{Requirements for screening, recommended frequency}

As we have already stated, a routine dental examination should include an examination of the periodontal tissues. The BPE (Basic Periodontal Examination) is an efficient way of doing this and Table 1 shows the codes that are recorded for each sextant. ${ }^{3}$ The “*' category recognises complex clinical situations, namely the involvement of a furcation or where recession and probing depth give an attachment loss level of $7 \mathrm{~mm}$ or more.

The findings of the BPE should be communicated to the patient, documented and acted upon. Other than a score of 0 , each score represents a level of disease that should be treated. A score of ' 1 ' is recorded where there is no pocketing greater than $3 \mathrm{~mm}$, but where there is bleeding on probing. This represents gingivitis, and the patient should be offered appropriate oral hygiene instruction. A code of ' 2 ' is gingivitis in the presence of local factors such as calculus or a poor restoration margin. The local factor should be addressed and oral hygiene instruction offered. The management of the problem highlighted by the 

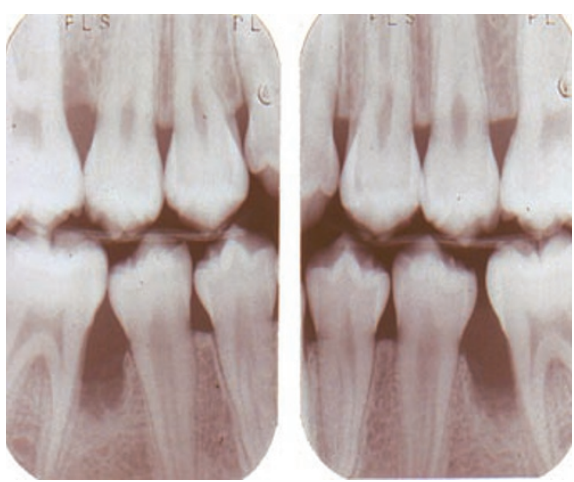

Fig. 3 Advanced periodontal bone loss in a 15-year-old boy

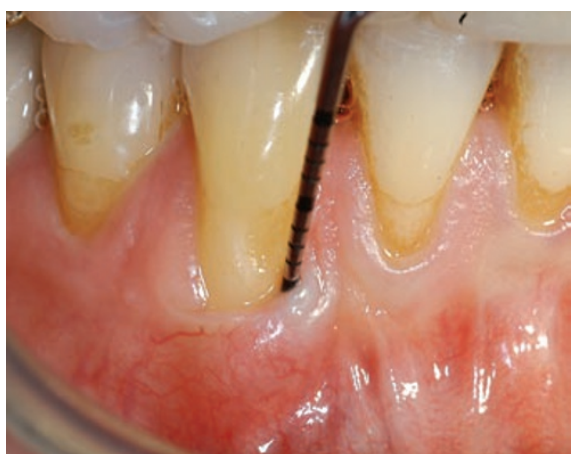

Fig. 4 Probing depth will be recorded as $4 \mathrm{~mm}$. This underestimates the actual level of attachment loss

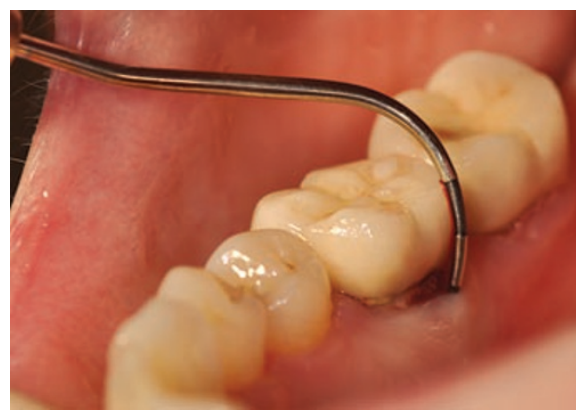

Fig. 5 A furcation probe in situ

BPE should be documented in the notes. When scores of ' 3 ', '4' or '*' are recorded, these indicate attachment loss and a further detailed periodontal examination is required, in the involved sextant in the case of a ' 3 ' and of the full mouth in the case of a ' 4 ' or '*. Since the BPE, once practised, is a quick examination, it should be incorporated as part of the routine dental examination. It must be stressed that the BPE is designed for screening and must be acted upon. It does not give sufficient information to make a diagnosis or to allow longitudinal monitoring of disease progression or the response to treatment.

The interval between periodontal examinations depends very much on patient needs. ${ }^{4}$ A patient who is not periodontally susceptible may require infrequent examinations. Unfortunately the patient's susceptibility can often only be judged retrospectively. There are occasions where periodontal attachment loss can occur in a relatively short timeframe. In some patients, risk factors might be present that help to predict such a breakdown, including poorly controlled diabetes or smoking. There is a small group of the population susceptible to the aggressive forms of periodontal disease, both localised and generalised. Figure 3 presents the radiographs of a 15-year-old boy with localised aggressive periodontitis. These patients can undergo extensive bone loss in a very short period of time.

\section{DETAILED PERIODONTAL EXAMINATION}

Once significant periodontal attachment loss has been diagnosed, the clinician should perform a full periodontal examination. The full periodontal examination records a baseline clinical picture for the patient. This is essential for assessing the response to treatment, and also for long-term comparison in the assessment of future periodontal breakdown. The full dental and periodontal examination, along with radiographic examination, will give the clinician the information required to treatment plan cases of moderate to advanced disease, or where there are also significant restorative problems.

The full periodontal assessment should include a review of the oral hygiene. At this stage in treatment, a detailed plaque chart is not necessarily required, rather a view of the patient's need for oral hygiene instruction and their response to it. Later stages in treatment may well require a detailed objective assessment of the oral hygiene in order that the clinician can decide on what treatment options are likely to succeed. Surgery, for example, is unlikely to succeed in all but the short-term when performed on a patient without adequate plaque control.

Bleeding scores have become an essential part of the periodontal examination. At the initial stage, the bleeding following probing may well be from the superficial tissues as a result of marginal inflammation (gingivitis). Following a well performed course of nonsurgical therapy and where the patient has a good level of plaque control, the bleeding may be originating from the depth of the pocket, indicating residual inflammation at the base of the pocket. This is considered to be a risk factor for disease progression and such bleeding may be an indication for further treatment.

Probing depths will need to be recorded, as these indicate sites that require treatment and also allow long-term comparisons for monitoring disease progression. Where there is gingival recession, this should also be recorded to give an indication of the actual amount of attachment loss (Fig. 4). Patients who have thin tissues may develop recession rather than pocketing as their periodontal disease progresses. In such cases, the probing depth may remain shallow and constant in the presence of continued attachment loss. Such situations will only be obvious to the clinician where the increasing recession can be monitored.

Individual tooth mobility scores should be recorded. Mobility may reflect the extent of alveolar bone loss, but mobility may also be a reflection of occlusal forces (including parafunction and interferences). The occlusion should be examined for any such interferences and a record made. This can then be reviewed with radiographs to build up a picture of the processes leading to the increased mobility. Other aspects of occlusion that are important include assessing the risk of teeth drifting, such as the position of the lower lip in relation to the upper incisors, parafunction and loss of posterior support.

Furcation involvements will have a significant influence on the periodontal prognosis of a tooth. Furcation involvements should be recorded and where it is possible, horizontal measurements of involvement (in $\mathrm{mm}$ ) can help in monitoring progression (Fig. 5). Recognition of these limitations to treatment response helps to predict outcomes and to achieve realistic expectations from treatment.

\section{SPECIAL TESTS}

Following the detailed periodontal examination, the clinician is in a position to decide what special tests are required.

\section{Radiographs}

The decision of what radiographs to take will depend on the extent and distribution of the periodontal disease from the clinical examination. Overall, intraoral 
films are best for periodontal diagnosis and caries. However, a balance needs to be struck between diagnostic value and radiation exposure. The FGDP guidelines are helpful in this respect. ${ }^{5}$ Well aligned bitewing radiographs will allow assessment of early levels of bone loss affecting the posterior teeth, and vertical bitewings allow assessment of early to moderate levels of bone loss. Periapical radiographs will show advanced levels of attachment loss, as well as other features, such as the root anatomy, possible furcation involvements, apical radiolucencies and widened ligaments. These will help in the diagnosis of the pathological and physiological processes that are influencing the clinical picture and will all influence the periodontal prognosis of an individual tooth. Further to this, the clinician must also consider the endodontic and restorative status of the tooth to ascertain the overall prognosis of the tooth.

Good quality panoramic radiographs have the advantage of showing the entire mouth. The nature of panoramic radiography may result in a lack of fine detail that is useful in the assessment and monitoring of periodontal disease. The information shown represents a focal trough and information outside this will not be shown in detail and there is also a degree of magnification.

The decision on which areas to radiograph will depend on the clinical examination. Periapical radiographs should be taken with a film-holder and paralleling technique since bisected angle radiographs, however well taken, distort the image. This gives a picture that is very close to actual size, and also improves the reproducibility. A degree of reproducibility in position and angulation of radiographs is extremely useful in the long-term monitoring of periodontal disease.

Follow-up radiographs may be taken in the long-term depending on clinical need. For instance, these might be needed to assess stability of the bone levels and look for areas of continued bone loss. Such radiographs should follow a periodontal examination and be based on a perceived need for the additional information that the radiograph will yield. Acute problems, such as periodontal abscesses, rapid development of deep probing sites or sudden increases in mobility will require radiographic examination to aid diagnosis.

When treating periodontal disease, the initial changes in probing depth following conventional treatment, both surgically and non-surgically, are mostly due to changes in the soft tissues. Obviously, further radiographs are unlikely to show significant changes from baseline and cannot be justified for at least a year, unless major changes occur. Small clinical changes of $1 \mathrm{~mm}$ may represent probing error rather than actual deterioration, and radiographic examination is unlikely to be of diagnostic benefit.

\section{Vitality testing}

Endodontic lesions can, on occasion, mimic periodontal lesions. Periapical abscesses can drain along the periodontal ligament and present as a periodontal pocket probing to the apex (Fig. 6). Therefore, vitality testing is recommended for the following situations:

1. Attachment loss approaching the root apex

2. Deep sites unresponsive to nonsurgical treatment

3. Periodontal pockets associated with deep furcation involvements

4. Periodontal pockets associated with heavily restored teeth.

Perio-endo lesions can be difficult to diagnose, as can situations where there are vertical fractures (Fig. 7). Correct treatment will depend on the correct diagnosis, and therefore it is essential that the clinician gathers all possible information to help with said diagnosis. Where there is still doubt over the underlying cause, the least invasive treatment option should be considered first, as a failure to respond may aid diagnosis.

\section{PROGNOSIS}

When all the appropriate data has been gathered, the first step is to make a diagnosis. The diagnosis should be relayed to the patient with an indication of its significance. The diagnosis may also help with the decision making and treatment planning. For example, a diagnosis of an aggressive form of the disease may mean that adjunctive systemic antibiotics will benefit the patient during non-surgical treatment. ${ }^{6}$ For periodontal disease, the

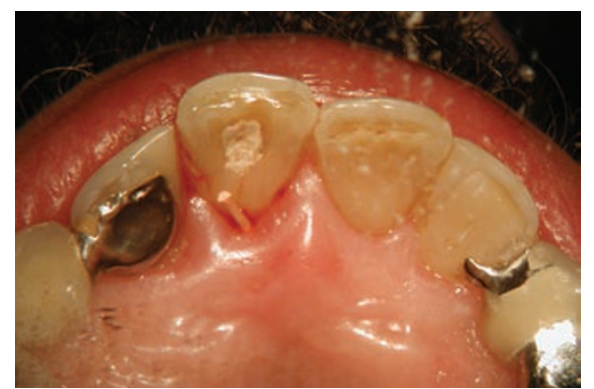

Fig. 6 An endodontic sinus draining along the periodontal ligament may mimic a periodontal pocket

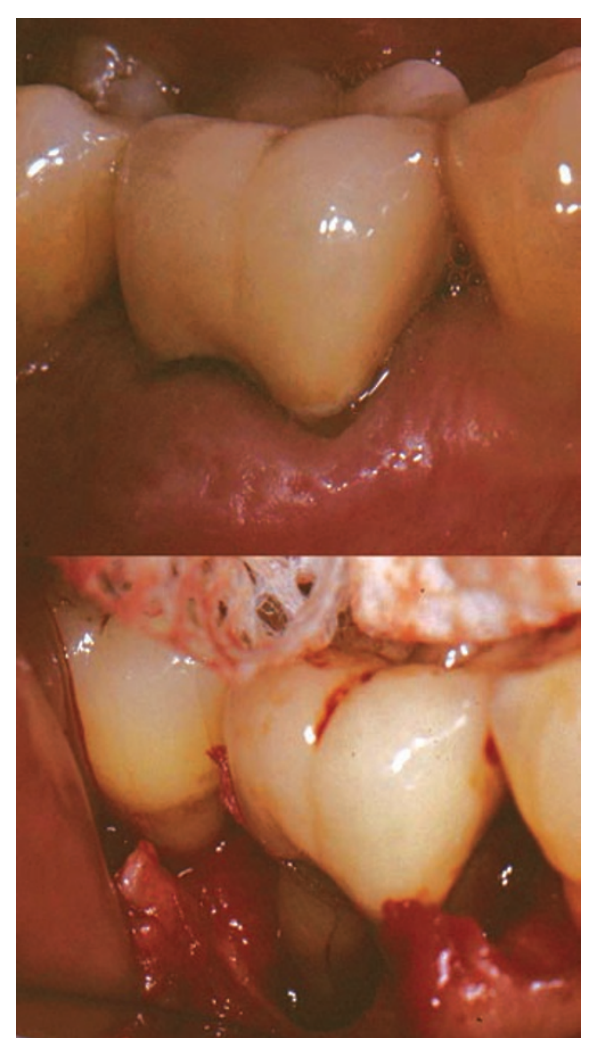

Fig. 7 Rapid periodontal breakdown occurred on the distal root and into the furcation. Surgical exploration revealed the vertical fracture on the distal root

prognosis may be patient-based, or done for each tooth individually, or both.

The prognosis for the patient will take into account systemic risk factors such as smoking or susceptibility. The susceptibility of the patient can be considered as the severity of the disease in the context of how old the patient is. The longterm outcome is also highly reliant on the patient's motivation, their ability to perform an adequate level of oral hygiene and their willingness to maintain this in the long-term. These factors cannot usually be judged early on in treatment, and the patient prognosis may be adjusted later on when such factors become more apparent. 
A tooth-by-tooth prognosis is extremely important when dealing with cases that involve more than one branch of dentistry. Where teeth need to be extracted and replaced, or where teeth have endodontic or restorative issues, treatment planning can become a complex and confusing process. To approach this logically, a decision needs to be taken as to whether or not individual teeth are likely to survive in the short-, medium- and long-term. Obviously a patient will not be happy if their new bridge fails after a short period because the abutments had untreatable periodontal disease or endodontic complications. Sometimes it is appropriate to use compromised teeth, for instance as part of a transitional treatment plan or if the prognosis is reasonably favourable but not yet clear. In this situation, the patient must be fully aware of the risks of failure and agree to accept the compromise after an explanation of why such a plan is being considered, and what the options are. This does not give the dentist the right to undertake inappropriate treatment, just because a patient has agreed to it.

\section{TREATMENT PLANNING}

Treatment planning may be a relatively simple process or it may be complex, depending on the degree of the periodontal attachment loss and the extent of other restorative dental problems that need to be considered. Obviously the more problems that need to be addressed, the more possible options there are to be considered. The nature of the treatment plan may be influenced by the patient's presenting complaints and their specific requirements. That said, patients must be made aware of all reasonable options and the advantages and disadvantages involved.

There are a number of adjunctive antibiotic and antiseptic devices that are commercially available for use in the treatment of periodontal disease. The decision to use such products lies with the clinician, within the context of informed consent. What should be stressed is that these products are designed to be used as adjuncts to properly performed nonsurgical periodontal treatment. They are unlikely to be of any benefit to the patient in all but the short-term in the absence of adequate supragingival and subgingival debridement and an appropriate level of patient-centred plaque control. Systemic antibiotics may useful in the management of acute periodontal conditions or in aggressive disease where they are used as an adjunct to non-surgical treatment. There is no evidence of benefit in using systemic antibiotics in the management of early-moderate chronic periodontitis. ${ }^{6}$

One key issue with the treatment of moderate to advanced periodontal disease is the relative uncertainty of the outcome of treatment. Initial treatment will almost invariably start with a course of nonsurgical therapy. This may be undertaken with the knowledge that surgery is likely. However, the decision to proceed with surgery can only be taken at the reassessment stage, based on the need still being present and on several factors being favourable, such as an appropriate level of oral hygiene. Patients should be informed of this and the reasons for initial uncertainty about the treatment needs explained.

The initial uncertainty about teeth with a questionable periodontal prognosis may also affect treatment planning. Teeth with such a prognosis may also have significant restorative requirements. However, the medium- to long-term prognosis may be uncertain until after periodontal treatment. In such cases primary disease such as caries or apical pathology should be addressed, with the deferment of complex and potentially expensive treatments until after a favourable response to periodontal treatment has been seen. The provision of a provisional bridge, for example, where one or more of the abutment teeth has a questionable periodontal prognosis will reduce the initial cost to a paying patient. The patient is less likely to be dissatisfied should the supporting teeth prove periodontally untreatable in a relatively short time. If the periodontal prognosis of the abutment teeth looks favourable following the periodontal treatment, then the permanent bridge can be made with more confidence about its longevity.

\section{Restorative dentistry}

Restorations can contribute to periodontal disease where they act as plaque retentive factors. Subgingival margins and overhanging or deficient margins in particular may contribute to localised

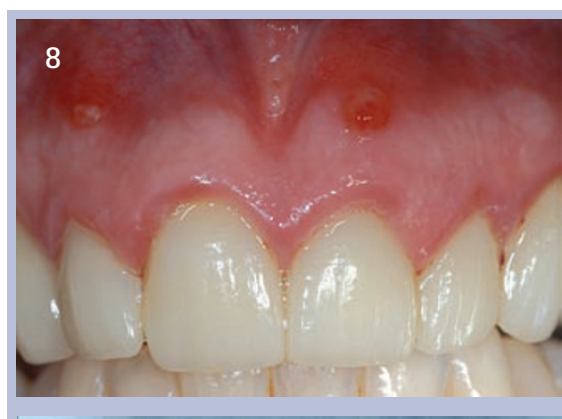

9

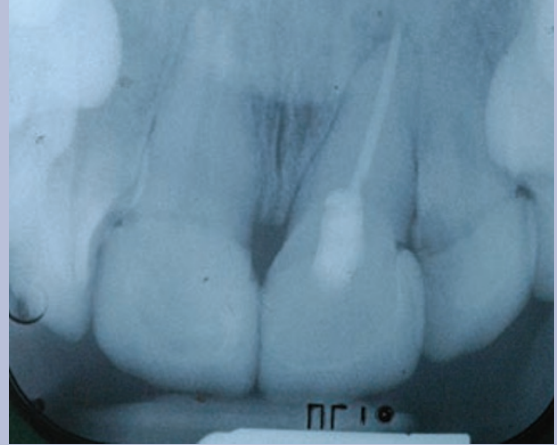

Figs 8 and 9 Poorly fitting veneers with subgingival margins leading to marginal inflammation. Over-preparation has also led to a loss of vitality of 12 and 21

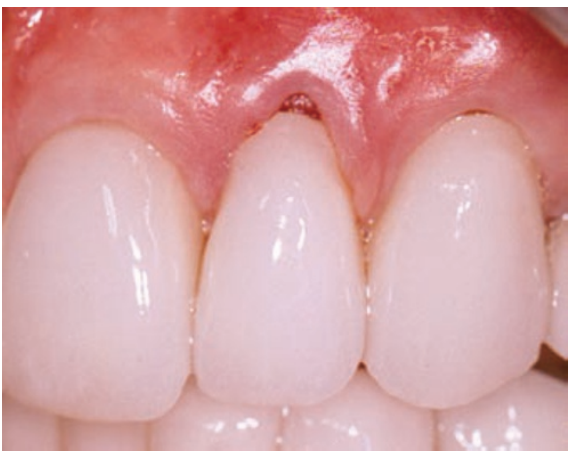

Fig. 10 Subgingival veneer margin in thin tissues may lead to gingival recession

inflammation and attachment loss (Figs 8 and 9). Wherever possible, margins should be properly finished and supragingival.

Invasion of the biologic width will result in an apical shift in the attachment apparatus. Where this is associated with thick gingival tissues, there will be an associated increase in probing depth. This may predispose to a progressive attachment loss in the susceptible patient. Where the tissues are thin, the patient is likely to develop localised gingival recession. While this is unlikely to progress, it might result in exposure of the restoration margin, which may be unacceptable to a patient in the aesthetic zone (Fig. 10). Recession might also occur where localised inflammation is not resolved before placement of the restoration. Placement of 
restorations under such circumstances is more likely to result in inadequate margins due to moisture contamination during the impression taking or bonding. If the patient does manage to control the gingivitis, then the subsequent gingival shrinkage may result in exposure of the margins. Margins should be finished supragingivally or just within the sulcus to allow adequate cleaning when aesthetics is critical.

\section{Implant dentistry}

The exact nature of the interaction between periodontal disease and dental implants is not clear, although current best evidence suggests that in patients successfully treated for periodontitis, there is an increased risk of implant failure and biological complications. ${ }^{7}$ In view of the evidence, patients should be warned about the risk. It is clearly unacceptable to place implants into a mouth that has untreated or inadequately treated periodontal disease.

\section{Alternatives - longevity and cost}

When formulating a treatment plan for an advanced periodontal case or a complex restorative case, there may be many alternative approaches. As we have mentioned before, a patient must be aware of all reasonable alternatives and options before accepting a treatment plan and consenting to treatment. The various pros and cons, risks and benefits, cost and long-term consequences should be explained.

\section{PERIODONTAL PREVENTION AND TREATMENT}

\section{Education}

It would seem obvious that once a patient has been diagnosed with a condition, they should be informed of the nature and significance of that condition. In the case of chronic periodontal disease, successful treatment relies very heavily on patient motivation and adherence to self-care. Susceptible patients will be expected to spend a significant time each day undertaking a thorough cleaning regime. It is unreasonable to expect the patient to perform such tasks without understanding its importance.

Periodontal treatment plans can be expensive and involve many appointments, some of which may be relatively invasive. For patients to agree to such treatment, they must understand why the treatment is structured in the way that it is. Definitive treatment planning may not be possible at the outset. The prognosis of certain teeth and the patient in general may only become apparent after the response to the stages of treatment can be assessed. In such a case, it is important that the patient understands this at the outset. Patients who are unaware of this may be unpleasantly surprised after a course of invasive treatment, if they are only told then that they need further treatment.

\section{Oral hygiene}

Effective patient oral hygiene is one of the key factors in the successful treatment of chronic periodontitis. The long-term success of periodontal treatment, and in particular patients who are overtly susceptible, relies very much on them achieving and maintaining high standards of oral hygiene in the long-term.

Effective oral hygiene is the responsibility of the patient. However, the dental team's role is to provide appropriate advice and training throughout the period of care. This responsibility includes assessment of:

1. Effectiveness of existing oral hygiene

2. The need for coaching and advice

3. The provision of appropriate advice and coaching including teaching of techniques and selection of aids.

Careful documentation will facilitate ongoing care by ensuring that all members of the dental team are informed about the patient's care (and choices) to date. The patient's reaction to such advice should also be recorded. Similarly, the patient should be advised of the level of their oral hygiene and also be educated as to why their homecare is such an essential part of effective periodontal treatment.

\section{Patients who have inadequate oral hygiene, despite adequate advice and coaching}

Complex treatment such as periodontal surgery is contra-indicated in the presence of inadequate plaque control. Before embarking on such treatment, patients should have proven to be motivated and able in their cleaning. Ideally this should be assessed and recorded by means of an objective measure such as a percentage plaque score.

Where patients fail to achieve an adequate level of oral hygiene, either through a lack of interest or ability, then the relevance of this must be explained clearly to the patient and the conversation documented in the notes. Such patients may benefit from regular periodontal maintenance where regular professional plaque control may, in part, compensate for the patient's inadequate level of oral hygiene.

\section{Smoking}

There is an established link between smoking and chronic periodontitis. Smokers experience increased amounts of attachment loss and respond less well to treatment. True 'refractory periodontitis', that is chronic periodontitis that does not respond to well performed conventional therapy, is rare. The majority of patients who do not respond to treatment in the long-term, in spite of adequate professional and homecare, are smokers.

Periodontal patients should be informed of the implications of their smoking habit and the effect that it will have on treatment and prognosis. Smoking cessation advice should be given. Current best practice is to refer patients interested in quitting for specialist advice such as NHS stop smoking clinics unless the dental team possesses these specialist skills. ${ }^{8}$

\section{Non-surgical treatment}

As with any clinical procedure, the clinician must be competent and suitably equipped for the job in hand. Sickle scalers are not designed for subgingival use as by definition they have a pointed end which can damage the gingival tissues. Periodontal curettes have a rounded end and are therefore suitable for subgingival use. No single curette can access all sites in a quadrant due to the design of the working ends. The clinician should have the appropriate instruments to be able to adequately access all the involved root surfaces.

Before non-surgical therapy, patients should be warned of likely side effects. The most common include:

- Recession (particularly the development of black triangles)

- Dentine sensitivity which is usually mild and responsive to fluoride toothpastes and mouthrinses

- Food packing between teeth.

Furthermore, it might be helpful to warn patients of a possible increase in bleeding 

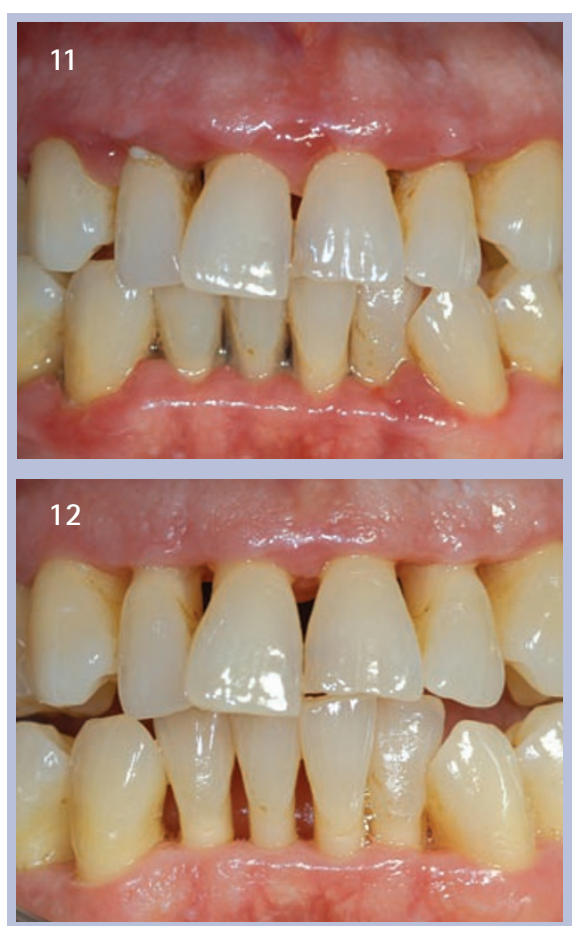

Figs 11 and 12 Soft tissue changes following non-surgical periodontal treatment

and tenderness at the commencement of thorough oral hygiene and scaling.

Non-surgical therapy may result in a period of dentine sensitivity following the removal of root surface deposits and subsequent gingival recession. Patients should be warned of this and encouraged to reattend should this become problematic. Where patients have particularly inflamed tissues, often associated with significant plaque retentive factors, then there is scope for significant amounts of recession to occur following non-surgical therapy. This may result in an aesthetic change, such as the creation of 'black triangles' between the teeth due to loss of the papillae (Figs 11 and 12). Patients should be warned of this before embarking on treatment.

\section{Role of the dental hygienist/therapist}

Any course of dental treatment should start with a full mouth assessment by a dentist. This should result in a diagnosis and treatment plan. If the treatment plan includes a referral to a dental hygienist (or therapist) then there is a shared responsibility for that patient. It is therefore imperative that both dental professionals understand their role in the management of that individual patient, and that communication between those profes- sionals ensures that the patient receives proper treatment.

When working with a hygienist, the dentist should explain exactly what is required. The current curricula frameworks produced by the General Dental Council as guidelines for the training of dental hygienists suggest that hygienists should, among other things, be able to:

- Be competent at providing preventive oral care for the individual patient who presents with dental caries, periodontal disease and tooth wear

- Be competent at completing a periodontal examination and charting

- Be competent at supragingival and subgingival scaling and root debridement, using both powered and manual instrumentation, and in stain removal and prophylaxis

- Be competent in the use of appropriate antimicrobial therapy in the management of plaque related diseases. ${ }^{9}$

When referring the patient to the hygienist, the dentist must be clear what is expected from the hygienist, what aspects of the periodontal treatment plan are to be undertaken by the hygienist, and what will be undertaken by the dentist. The role of the hygienist must fall within the scope of their competence.

Once the patient has been referred to the hygienist, the dentist can ask the hygienist to set the recall intervals, defined as how often the patient should return to be seen by a member of the dental team. The dentist, however, should also set a reassessment date, when the patient must return to be seen by a dentist for a full-mouth examination and treatment plan.

The General Dental Council, in its document 'Principles of dental team working, ${ }^{10}$ states that 'all members of the dental team who have to register with us are individually responsible and accountable for their own actions and for the treatment processes which they carry out.' However, the dentist does take on overall responsibility for the care of the patient. While under the care of a hygienist, the hygienist has a responsibility to the patient to refer back to the dentist when it is appropriate and this should be defined by the dentist, as well as the procedure for doing this. This may be at the end of a defined treatment plan, but should also occur if the patient is not responding as would be expected.

A standard periodontal treatment plan, be it for a course of non-surgical or surgical treatment, will end with a reassessment. Since the supervising dentist is ultimately responsible for the patient's well-being, it would seem appropriate that such a reexamination is performed by, or in discussion with, the dentist. The next stage of patient care, be it further active treatment or maintenance, will then be planned by the dentist.

The NICE guideline on dental recall ${ }^{4}$ is specifically aimed at helping clinicians to decide on appropriate recall intervals between oral health reviews. It does not give guidance on the intervals between examinations relating to ongoing courses of treatment. This would include a course of periodontal treatment.

\section{Surgical treatment}

Periodontal surgery plays a role in achieving the main aim of periodontal treatment, which is long-term maintenance and prevention of continued attachment loss. This is facilitated by access to the root surfaces for visualised debridement, and by reshaping the hard and soft tissues to facilitate post-surgical maintenance, both professional and by the patient. Periodontal surgery does not represent a magic bullet in the treatment of chronic periodontitis. Its success will rely on a number of factors.

Periodontal surgery is destined for failure in the absence of immaculate oral hygiene by the patient and long-term periodontal maintenance. In almost all cases, periodontal surgery is only considered following the completion of a course of thorough non-surgical treatment. One of the key factors in this is establishing a proven level of patient homecare and a commitment to periodontal treatment. Sufficient time should also be allowed following the active phase of treatment to allow the response to the non-surgical treatment to be assessed. Surgical treatment can then be considered for sites that have not responded to the less invasive therapy. The non-surgical treatment will also improve the health of the superficial tissues, which facilitates the surgical phase, improving the soft tissue handling and reducing the amount of intra-operative bleeding. 
The initial extent of attachment loss will, to a degree, dictate the end result. Periodontal surgery may be aimed at pocket elimination where this achievable, or access for debridement. The latter may be indicated where deep persistent pocketing or complex root anatomy limits non-surgical debridement. Where the extent of the bone loss is excessive, periodontal surgery may be destined to fail and in such cases can lead to hastening the loss of the tooth. Obviously patients need to be aware of this before agreeing to undergo the discomfort and expense of such a procedure.

Conventional periodontal surgery has a resective component, be it excision of part of the soft tissue or bone reshaping by means of osteoplasty or ostectomy. This can result in a dramatic apical shift of the dento-gingival tissues resulting in gingival recession. Surgically created recession in the upper anterior region may be aesthetically unacceptable to the patient. A patient should certainly be warned about such recession before consenting to anterior periodontal surgery. There is evidence that repeated courses of non-surgical treatment may result in a similar end result, however this will occur over a relatively long timeframe. Since the change occurs gradually, the impact might be less dramatic

When undertaking periodontal surgery, patients should be given appropriate pre-operative warnings for oral surgical procedures. Risks of prolonged bleeding and infection are minimal with careful technique and appropriate postoperative instructions. Nerve damage is a remote risk unless with injudicious surgery to the lingual of the lower second and third molars and buccal to the lower premolars. Post-surgical pain is usually minimal, but will vary depending on the extent of the surgery, the time that the mucoperiosteal flaps are raised and the amount of exposed alveolar bone left after surgery. Patients should be warned of this and the appropriate analgesic advice given. A chlorhexidine rinse should be given to compensate for the inability to mechanically clean the surgical area in the immediate post-surgical phase. Patients should also be warned about sutures and also periodontal dressing if this is to be used.

One essential component of postoperative care will be professional plaque control during the period that self-performed plaque control is not possible or difficult. Professional care must assume this responsibility including plaque removal and modification of self-care methods.

\section{Regenerative materials}

Periodontal regenerative surgery utilises various surgical adjunctive materials with the aim of promoting regeneration of cementum, periodontal ligament and alveolar bone lost though periodontal disease. The initial protocol for 'guided tissue regeneration' utilised Gore-Tex membranes which were secured over periodontal bone defects beneath the mucoperiosteal flaps. More recently, other materials and approaches for periodontal regeneration have been researched and are now available for use in dental practice.

Resorbable membranes have the advantage that, unlike the Gore-Tex membranes, they do not need to be removed. Many resorbable membranes are made from synthetic materials, however a significant proportion are derived from bovine or porcine collagen. Bovine derived bone mineral derivatives are also commonly used, as are enamel matrix proteins which are extracted from young pigs. All these materials have been approved for medical use and should be considered safe. The decision, however, to insert any of these materials lies with the patient, and should be made by the patient with enough information regarding the moral and medical implications. Regenerative procedures are also technically challenging and can be unpredictable without very careful case selection. Patients should be aware of this before agreeing to treatment.

\section{Consequences of treatment - aesthetic and functional}

As we have mentioned previously, periodontal treatment can result in irreversible changes that can inconvenience the patient. Gingival recession may create an aesthetic issue for the patient, in particular the creation of 'black triangles' between the teeth where the papillae have been lost. Gingival recession in combination with root surface instrumentation often leads to dentine sensitivity. This can usually be controlled by local measures but on occasion can become persistent

\section{Periodontal maintenance/ supportive periodontal treatment}

Chronic periodontal disease is not cured and treatment is aimed at control. The parameters used to monitor periodontal disease are predominantly plaque control, probing depths and bleeding on probing. None of these are good predictors of sites at risk of attachment loss; however they are associated with an increased risk of attachment loss occurring.

Patients entering maintenance following the treatment of chronic periodontitis are likely to fall into one of two categories. The first are patients who have reached the end of active treatment and are considered 'treated'. These patients must be monitored and assisted in maintaining the posttreatment result. They require regular oral hygiene re-inforcement, regular supragingival and subgingival debridement, and close monitoring of the probing depths. Recurrence of increased probing depths will require further active treatment.

The second group are patients who are deemed untreatable. These may be patients who consistently fail to achieve an adequate standard of oral hygiene, either because they will not or cannot clean properly. Other patients may not be prepared to complete treatment for financial reasons or because they are not prepared to undergo complex periodontal treatments such as surgery. Such patients will benefit from regular maintenance, though this is aimed at slowing disease progression.

The interval between maintenance appointments should be tailored to the patient's need. Often after active treatment for chronic periodontitis, an initial three monthly recall interval is set. The reexamination date should also be set. The maintenance interval may be increased after periods of stability have been observed. If patients show signs of disease recurrence or attachment loss during maintenance, then they should be assessed and a new treatment plan formulated to address their problems.

\section{WHEN TO REFER}

When a clinician accepts a patient, he or she has a duty of care for that patient. The decision of whether to treat or whether to refer will depend on the clinician's ability, determined by their training and experience. If they undertake periodontal 
treatment, then they should be monitoring the response and reacting accordingly. If the patient does not respond in the expected manner, if they continue to lose attachment for example, then the treatment plan should be adjusted accordingly and this may include referral to a more experienced practitioner or specialist. The British Society of Periodontology has drawn up guidelines on patient referral in their document titled 'Referral policy and parameters of care. ${ }^{11}$

A BPE score of 3 or less should be treatable by means of relatively simple periodontal therapy. It is reasonable to expect a properly trained dentist to be able to manage such patients. That said, where there are aggravating factors or an unusual presentation, the involvement of a specialist may aid diagnosis and management.

A BPE score of 4 implies moderate to advanced disease. Whether or not to refer depends on the competence of the practitioner in managing the presenting patient. If the level of disease is beyond their ability or experience, then they have a duty of care to the patient to offer referral to someone who is suitably trained. The extent of the disease is not the only factor that should be considered. Risk factors should also be taken into account. Smokers may provide more challenges to treatment in the long-term and where there is advanced disease, early referral may be appropriate.

Aggressive disease, and in particular where this presents in people under 35 years of age, may also benefit from early referral. Such patients can lose bone at relatively rapid rates and may benefit from the care of someone experienced in their management. Obviously, the sooner such patients can be brought under control, the more likely they are to retain their teeth.

Patients who present with periodontal disease combined with significant levels of other dental disease can be demanding to treatment plan. Involvement of a specialist can help with formulating a suitable treatment plan.
1. Palmer R M, Floyd P D. A clinical guide to periodontology. 2nd ed. London: BDJ Books, 2003.

2. National Institute for Health and Clinical Excellence. Prophylaxis against infective endocarditis. Clinical guideline 64. London: NICE, 2008. http://guidance. nice.org.uk/CG64.

3. British Society of Periodontology. Periodontology in general dental practice in the United Kingdom. A policy statement. Leeds: British Society of Periodontology, 2001. http://www.bsperio.org.uk/ members/policy.pdf.

4. National Institute for Health and Clinical Excellence Dental recall. Recall interval between routine dental examinations. Clinical guideline 19. London: NICE, 2004. http://guidance.nice.org.uk/CG19.

5. Faculty of General Dental Practitioners (UK) Selection criteria for dental radiography. London: Faculty of General Dental Practitioners (UK), 2004.

6. Herrera D, Alonso B, Leon R, Roldan S, Sanz M. Antimicrobial therapy in periodontitis: the use of systemic antimicrobials against the subgingival biofilm. J Clin Periodonto/ 2008; 35(Suppl 8): 45-66.

7. Ong C T, Ivanovski $S$, Needleman I $G$ et al. Systematic review of implant outcomes in treated periodontitis subjects. J Clin Periodontol 2008; 35: 438-462.

8. National Institute for Health and Clinical Excellence. Brief interventions and referral for smoking cessation in primary care and other settings. Public health intervention guidance no. 1. London: NICE, 2006. http://quidance.nice.org.uk/PHI1/?c=296726.

9. General Dental Council. Developing the dental team. London: General Dental Council, 2004.

10. General Dental Council. Principles of dental team working. London: General Dental Council, 2009.

11. British Society of Periodontology. Referral policy and parameters of care. http://www.bsperio.org.uk/ members/referral.htm (accessed 31 March 2010). 